\title{
Long Term Outcome of Lupus Nephritis in SLE in Oman
}

DR TASNEEM AL RASHDI, DR JUMA AL KAABI

${ }^{1}$ [Department of Rheumatology, Sultan Qaboos University Hospital ]

\section{ABSTRACT}

Objective. To study the histopatholgical pattern of lupus nephritis, long
term outcome and identify the predictors of poor outcome among SLE

Methods. Tiss retrospective study included 94 patients diagnosed wi (a) score the type of lupus nephritis on renal biopsy. Patients were treated as (a) Meier survival curves and long rank test were used for survival aplysis. Results. 94 patients were included in the study with a female to male ratio or and the median duration of follow up was 8 years interquartile range [IQR Dand 7 patients died. up, hence, $50 \%$ achieved complete remission, $9.5 \%$ had partial remissio and $16 \%$ had active nepliritis. The overail henh survival at 5,10 and significant difference in survival distribution between histological class 1 and class $\mathrm{V}(\mathrm{p}=0.036)$. However, there is no difference in renal survival factors for poor ond regression analysis were onset to nephritis, elevated dsDNA and presence $\mathbb{C}$

Conclusion. our study is the first in Oman to address the outcome of lupus
nephritis in $\mathbf{O}$ mani populat reported in different parts of the worlds and the most commo dsDNA and infection were

\section{BACKGROUND}

Systemic lupus erythematous (SLE) is an autoimmune disease characterized by
the production of various autoantibodies directed against self-antigens. Tissue injuries and susbsequent organs damaged ensue resulting $i n$ various clinical $60 \%$ of patients with SLE will develop renal disease during the course of their $48 \%$ of patients at 5 years. The presentation of lupus nephritis (LN) is hiphly variable ranging from clinically silent nephritis to rapidly progressive disease with acute renal failure. In $3 \%$ to $6 \%$ of cases $\mathrm{LN}$ constitute the initial Despite the improved over all SLE survival over the last decays, patients with
LN Still succumb into chronic renal failure and death. Several studies Save those without renal involvement. Epidemiological studies in SLE with LN, have pointed out demograph
clinical and histological features associated with worse prognosis. the strong predictors of poor outcome is the histological pattern of $L N$. Diffuse proliferative (WHO class IV) of LN usually follows a progressive cours
to CRF without early and adeauate treatment. A 17\% 5-year survival without treatment has been reported. Further outcome analysis published betw
1990-2013, WHO class IV still leads to ESRD in 10-26\% of patients

OBJECTIVES

We have no published data about $L N$ in Omani patients yet. Thus, this study aims to provide information on the histological pattern, overall renal survival
and the predictors of poor outcome of lupus nephritis patients in Oman and

\section{RESULTS}

$$
\begin{aligned}
& \begin{array}{l}
\text { Table 1. Baseline characteristics of the patients } \\
\text { with lupus nephritis ( } \mathrm{n}=94)
\end{array} \\
& \begin{array}{lll}
\begin{array}{l}
\text { Varable } \\
\text { Sex }
\end{array} & \mathrm{n}(\%) & \text { Table } 1 . \\
\end{array}
\end{aligned}
$$

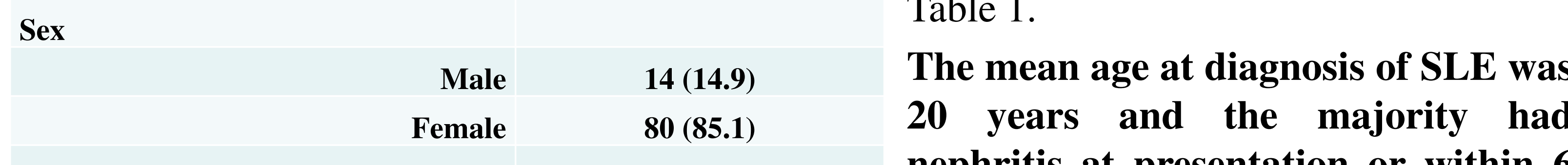

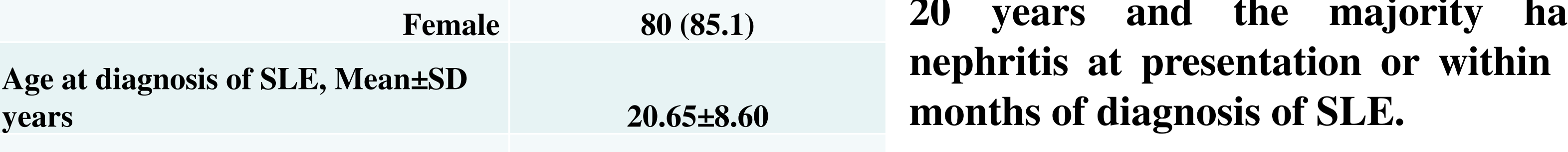

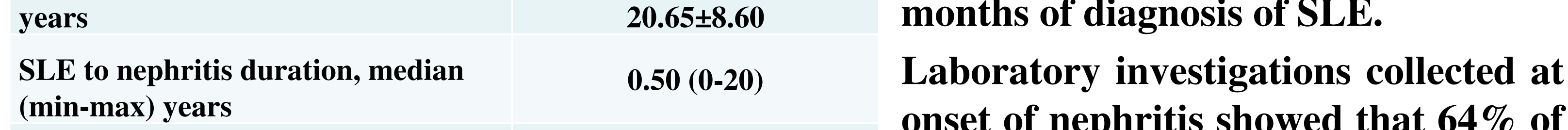

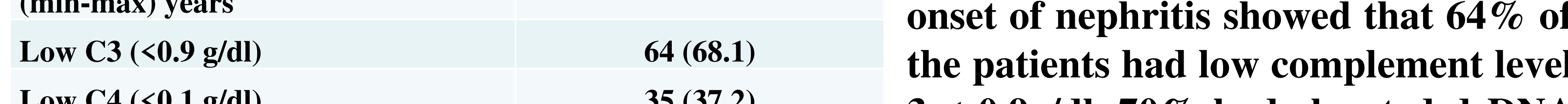

$$
\begin{aligned}
& 3<0.9 \mathrm{~g} / \mathrm{dll}, \mathbf{7 0 \%} \text { had elevated dsDNA }
\end{aligned}
$$

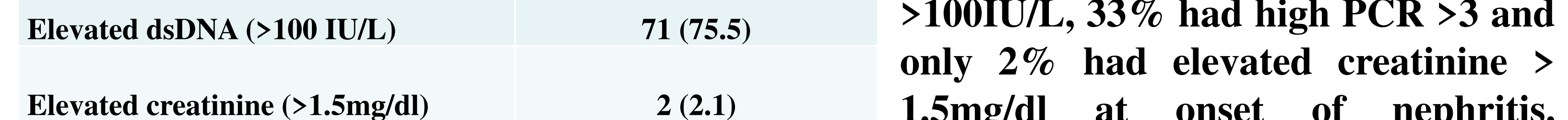

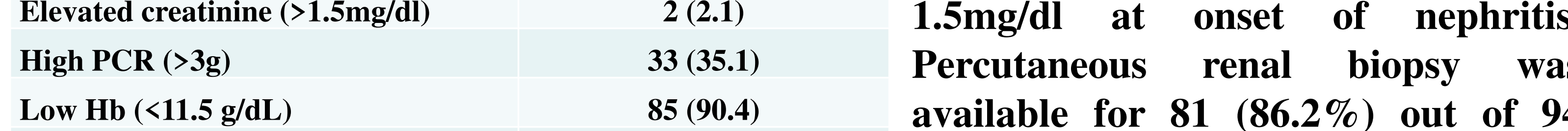

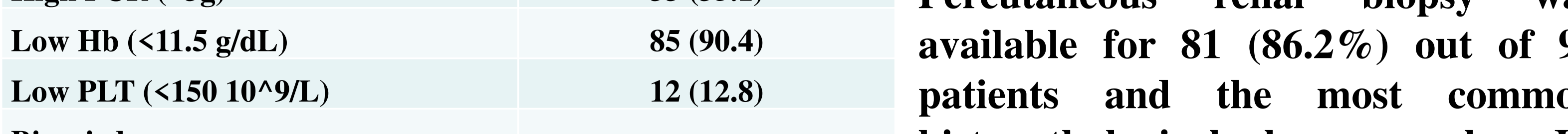

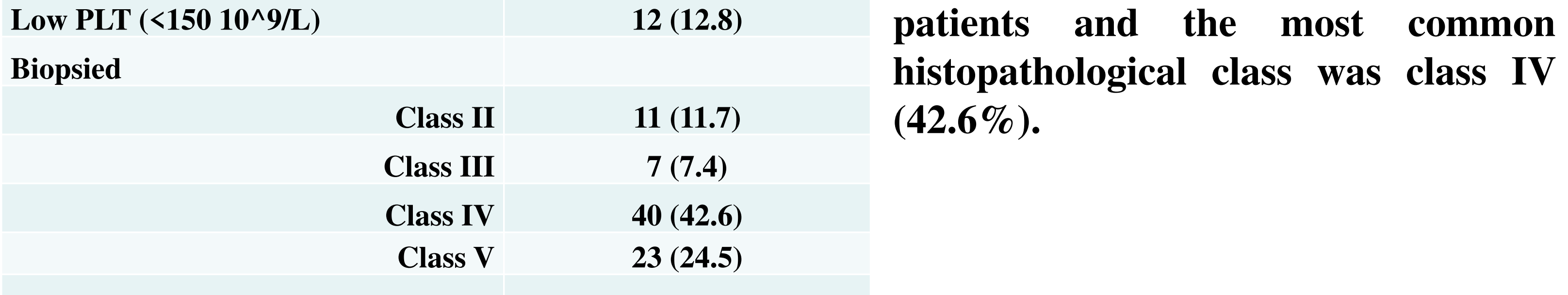

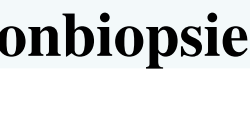

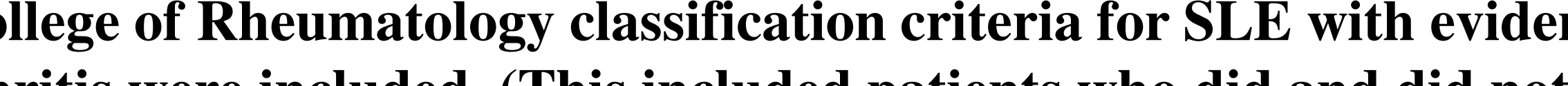

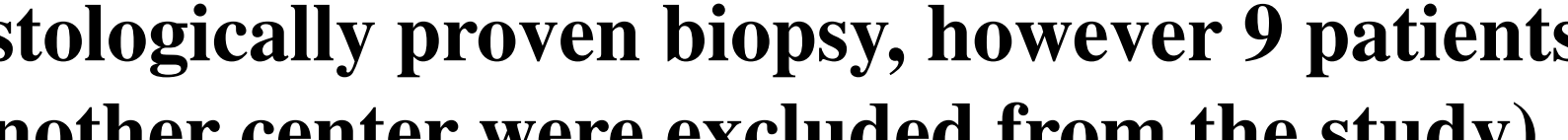

The response of treatment based on laboratory records on last follow up (urve for renal survival (CKD, ESRD and death were -Figure 1. Kaplan-Meier curve for renal survivial (CKD, ESRD and death were
considered as the hard end points). The overall renal survival at 5,10 and 15 year
was $869.9 \%, 738 \%$, and

Survival Functions
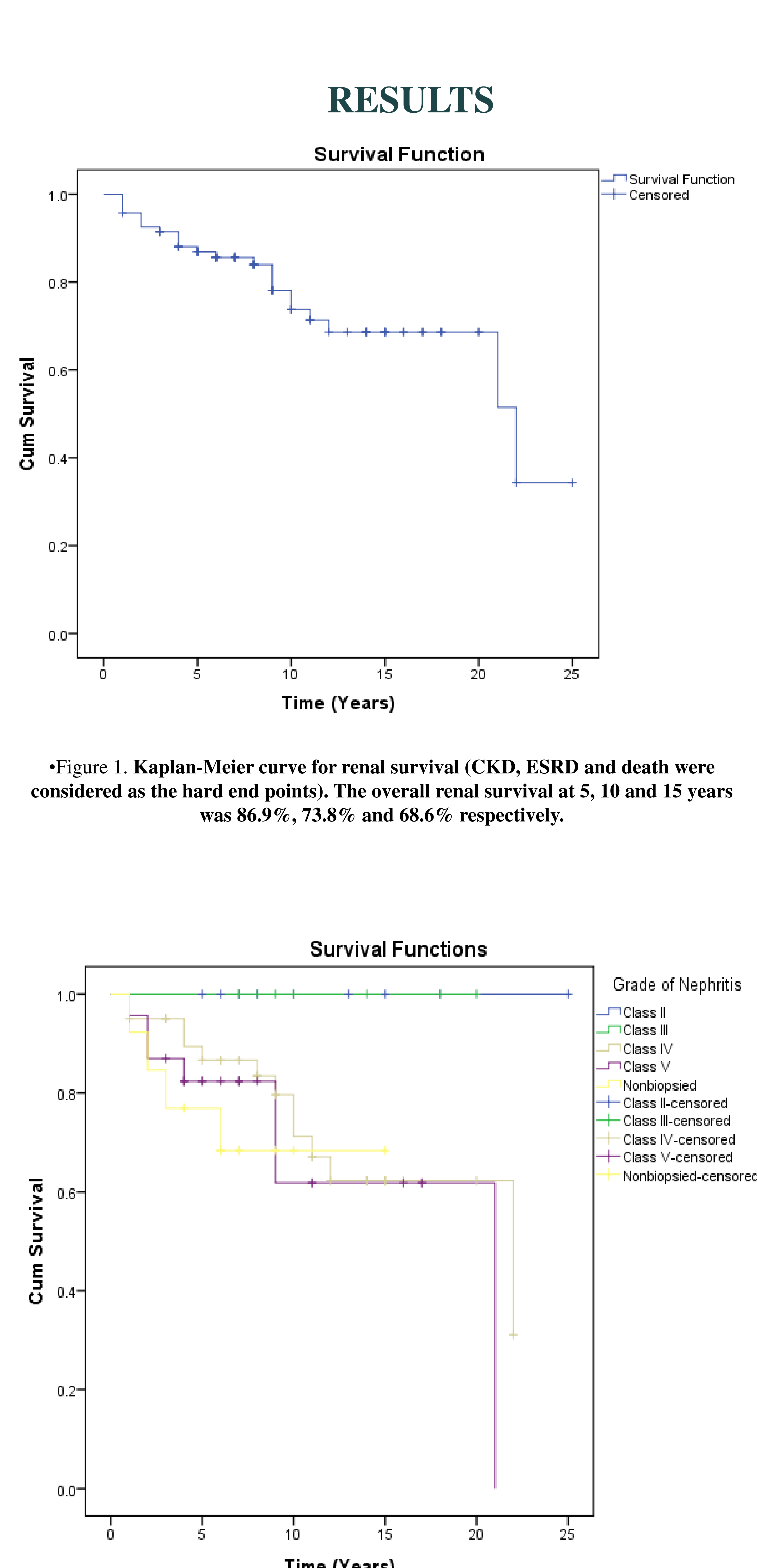

Figure 2. Kaplan-Meier curves for renal survival in the different histologic classes an non-biopsied patients. P< $<0.05$ by the log rank test between class II and V. There is
however, no difference in renal survival between apatients who had a renal biopsy and
non-biopsied patients.
RESULTS

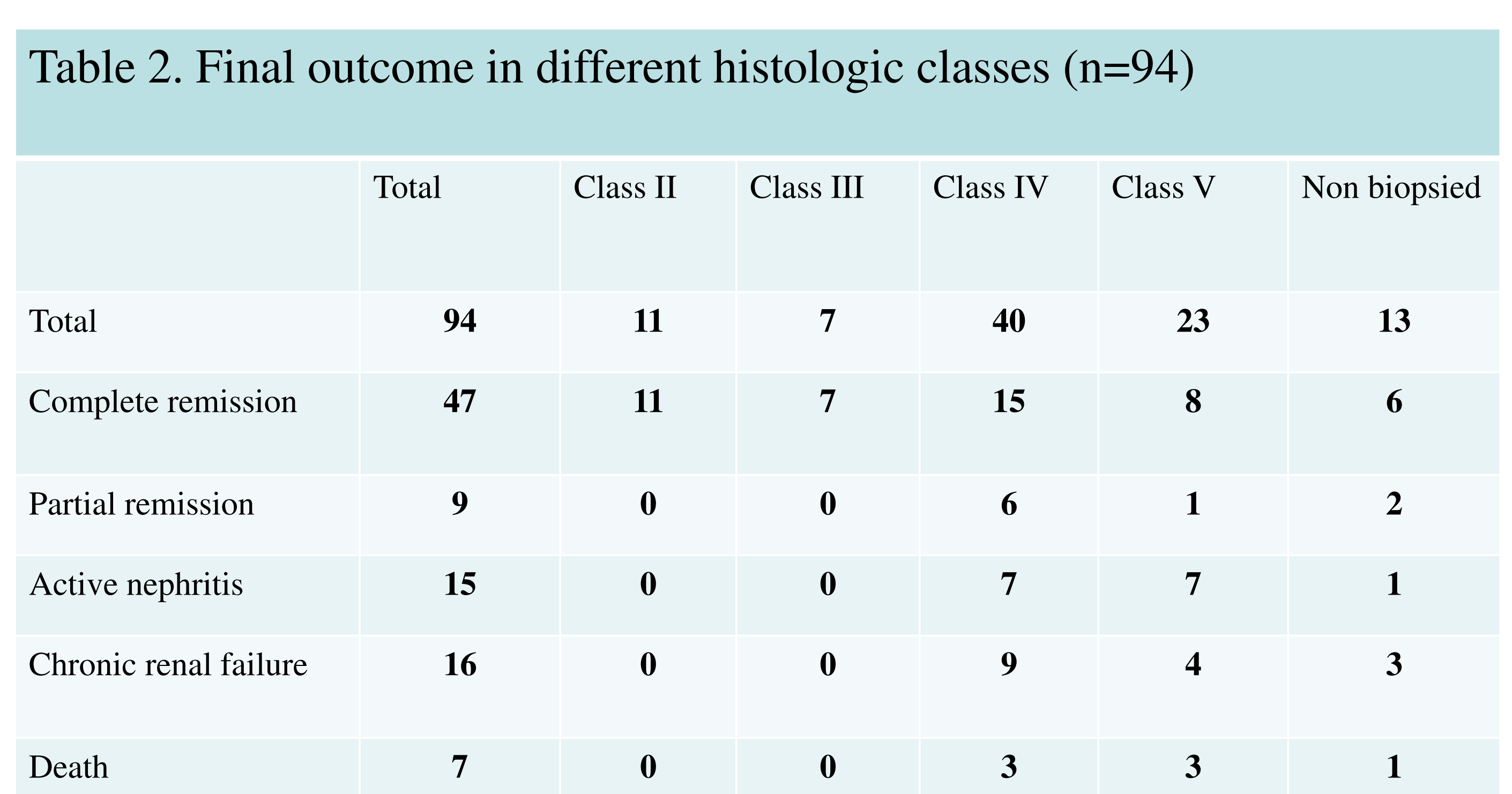

2able 2. The long-term outcome was calculated for all 94 patients and the media which 16 patients $(17 \%)$ developed CKD and 7 patients $(7.4 \%)$ died. Treattment response was based on PCR and creatinine level on last follow up, hence, $50 \%$
achieved complete remission, $9.5 \%$ had partial remission and $16 \%$ had active nephritis. All patients with class II and III LN achieved complete remiscion compared to patients with class IV and V LN whom around $35 \%$ achieved complete remission. 30\% of class IV lupus nephritis had poor outcome (defined as

\begin{tabular}{|c|c|}
\hline $\begin{array}{l}\text { Variable } \\
\text { Female sex } \\
\text { Age at diagnosis of SLE, years }\end{array}$ & $\begin{array}{c}\text { HR (95\% CD) } \\
0.329(0.116-0.930) \\
1.032(0.976-1.0991)\end{array}$ \\
\hline Onset to nephritits duration, years & $1.211(1.081-1.356)$ \\
\hline Low C3< $3<.9$ g/dil) & $2.414(0.696-8.378)$ \\
\hline Low C4(6.1 g/al) & $0.645(0.218-1.904)$ \\
\hline 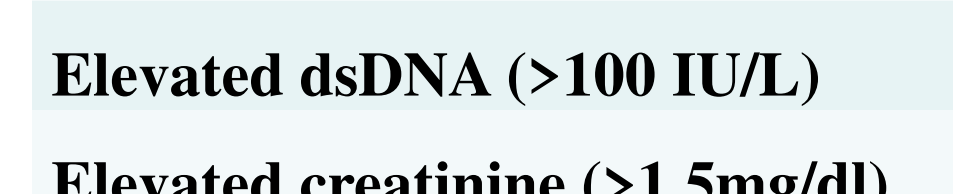 & $\begin{array}{l}8.107(1.247 .52 .711) \\
3.102(0315.320205\end{array}$ \\
\hline 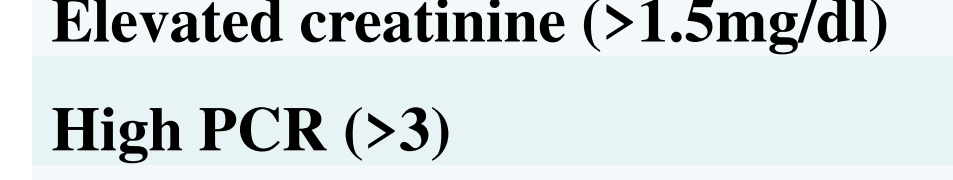 & 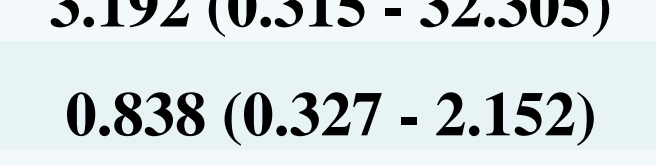 \\
\hline Low Hb (<1.5 g/dL) & $0.508(0.040-6.517)$ \\
\hline Low PLT ( 1150 1099/L) & $0.256(0.049-1.328)$ \\
\hline
\end{tabular}
Table 3. Cox regression analysis of risk factors leading to poor outcome (CKD, Table 3 . Risk factors for poor outcome on multivariate cox regression analysis
were onset to nephritis, elevated dsDNA and presence of infection. There is a 21.1\% increase in the expected HR relative to a year increase in onset of
to nephritis duration, holding all other factors constant $(\mathrm{p}=0.001)$. Th expected hazard is is. times higher in patients with elevated dsDNA $(p=0.028)$
and 4.9 times higher in patients who had an infection as compared to patients and 4.9 times higher in patients who had an infection as compared to patients

\section{CONCLUSIONS}

Our study is the first in Oman to address the outcome of lupus nephritis in different parts of the worlds and the most common histopathological class
was class IV. SLE to onset of nephritis duration, high dSDNA and infection 